
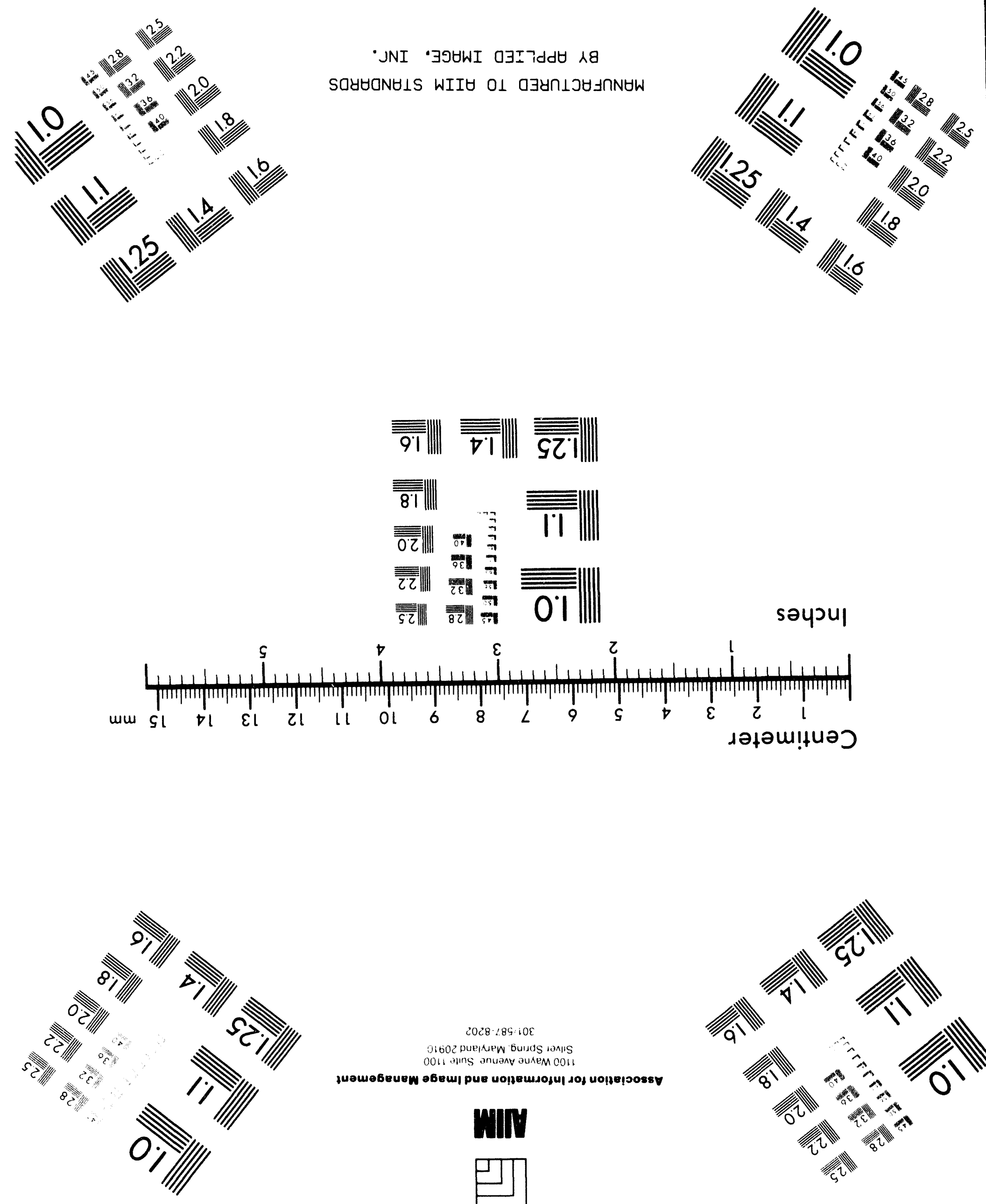

$2028 \cdot 285: 108$

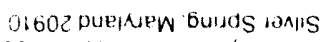

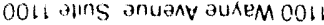

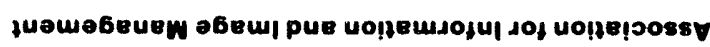
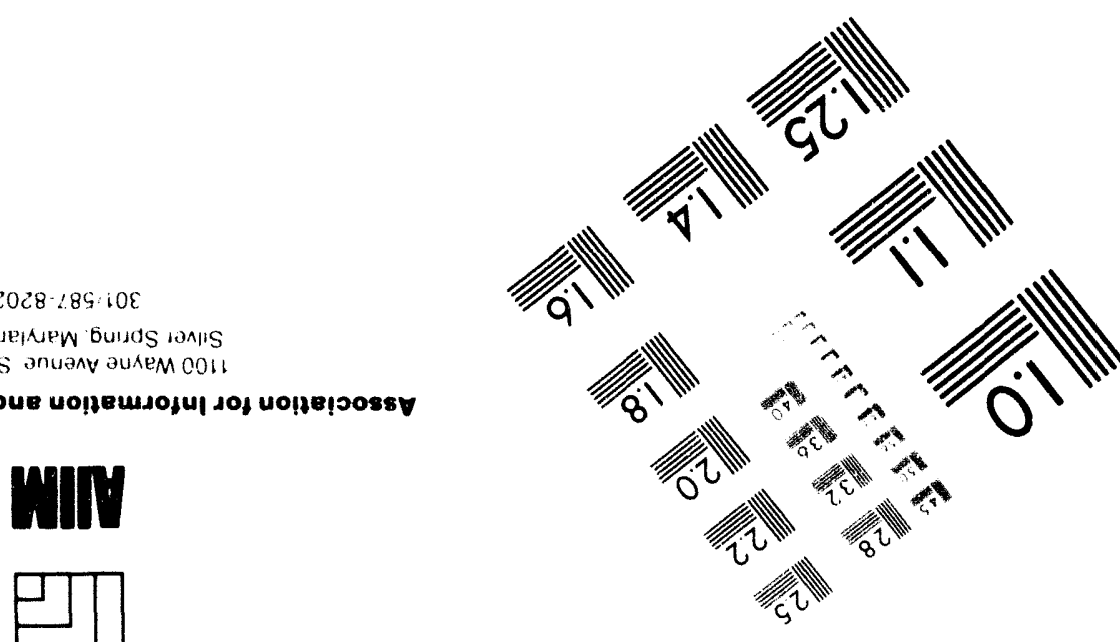

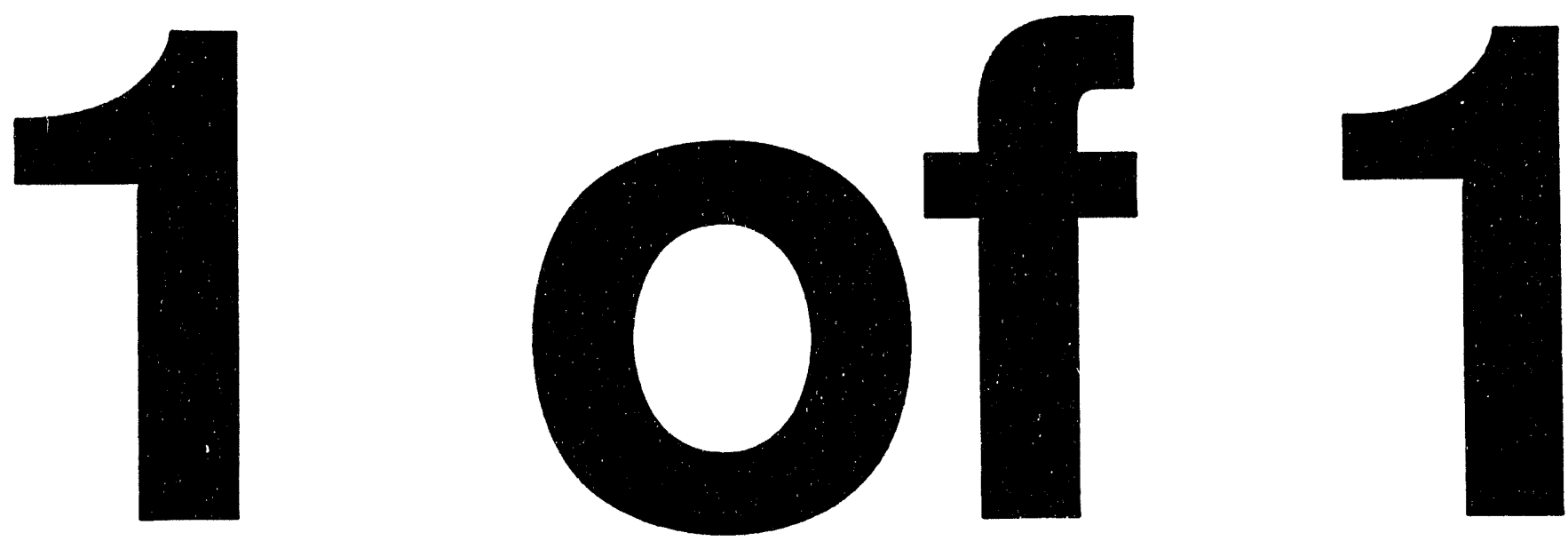
"The submitted manuscript has been authored by a contractor of the U.S. Government under contract No. DE-AC05-84OR21400. Accordingly, the U.S. Government retains a nonexclusive, royalty-free license to publish or reproduce the published form of this contribution, or allow others to do so, for U.S. Government purposes."

\title{
Comparison of the Folded Stripline and Stacked Stripline Concepts to the Folded Waveguide Launcher
}

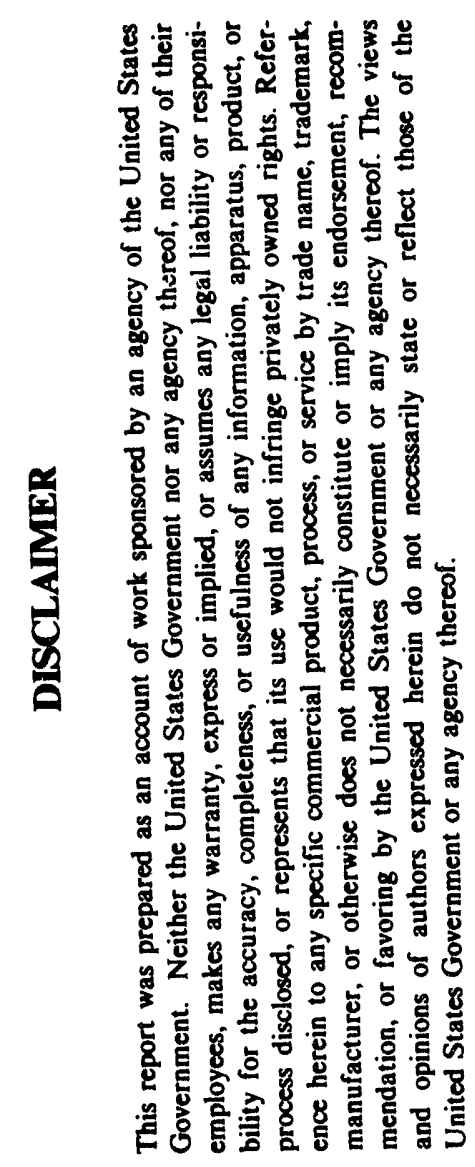

\author{
W. L. Gardner, J. B. O. Caughman, and D. J. Hoffman \\ Oak Ridge National Laboratory \\ Post Office Box 2009 \\ Oak Ridge, TN 37831-8071, USA \\ P. H. Probert \\ University of Wisconsin \\ Madison, WI 53706
}

\author{
Tenth Topical Conference on \\ Radio Frequency Power in Plasmas \\ Boston, MA \\ April 1 - 3, 1993
}

* Research managed by the Office of Fusion Energy, U.S. Department of Energy, under contract DE-AC05-84OR21400 with Martin Marietta Energy Systems, Inc. 


\title{
COMPARISON OF THE FOLDED STRIPLINE AND STACKED STRIPLINE
} CONCEPTS TO THE FOLDED WAVEGUIDE LAUNCHER *

\author{
W. L. Gardner, J. B. O. Caughman, and D. J. Hoffman \\ Oak Ridge National Laboratory, Oak Ridge, TN 37831 \\ P. H. Probert \\ University of Wisconsin, Madison, WI 53706
}

\begin{abstract}
Two new concepts are being developed as possible upgrades to the folded waveguide launcher. The folded stripline is a folded waveguide with an additional conductor positioned inside. The term stripline refers to the resemblance of the design to microwave microstrip line. The conductor provides support for TEM mode propagation, which eliminates cutoff and the nonlinear frequency dependence of the waveguide impedance and phase velocity. A natural extension to the folded stripline is the stacked stripline, which comprises several stacked, independent TEM waveguides. Initial measurements indicate that both concepts have better magnetic flux coupling than the folded waveguide.
\end{abstract}

\section{INTRODUCTION}

The folded waveguide cavity launcher, as depicted in Fig. 1, has been analyzed and tested for the last several years. ${ }^{1-5}$ The main advantages it offers over currently deployed loop-based designs are a higher coupled magnetic field (power) to the plasma and a low-output electrostatic field component that nearly eliminates the need for a Faraday shield. Its main disadvantages seem to be the large port size required for support of the waveguide fundamental mode, given reasonable power density and voltage handling limits, and the nonlinear frequency dependence of the waveguide impedance and phase velocity, which makes the launcher very sensitive to tuning. These disadvantages have led to two concepts based on supporting a TEM mode by introducing extra conductors to the basic cavity design.

\section{DESCRIPTION}

The concept known as the folded stripline merely adds a folded inner conductor to the basic folded waveguide structure, as shown in Fig. 1, and evolved from initially centering the inner conductor. The folded stripline behaves more like a two-conductor stripline to produce better coupling to the current-carrying straps. The current straps replace the polarizer plates to help channel current to create the correct magnetic field vector. The inner conductor is supported by the back cavity wall of the half-wave resonator. The preferred method of coupling If power to the cavity is through the center conductor of a coax cable connected to the inner conductor of the cavity. Tuning and matching can be done either externally to the cavity with standard coaxial tuning

\footnotetext{
*Research managed by the Office of Fusion Energy, U.S. Department of Energy, under contract DE-AC05-84OR21400 with Martin Marietta Energy Systems, Inc.
} 


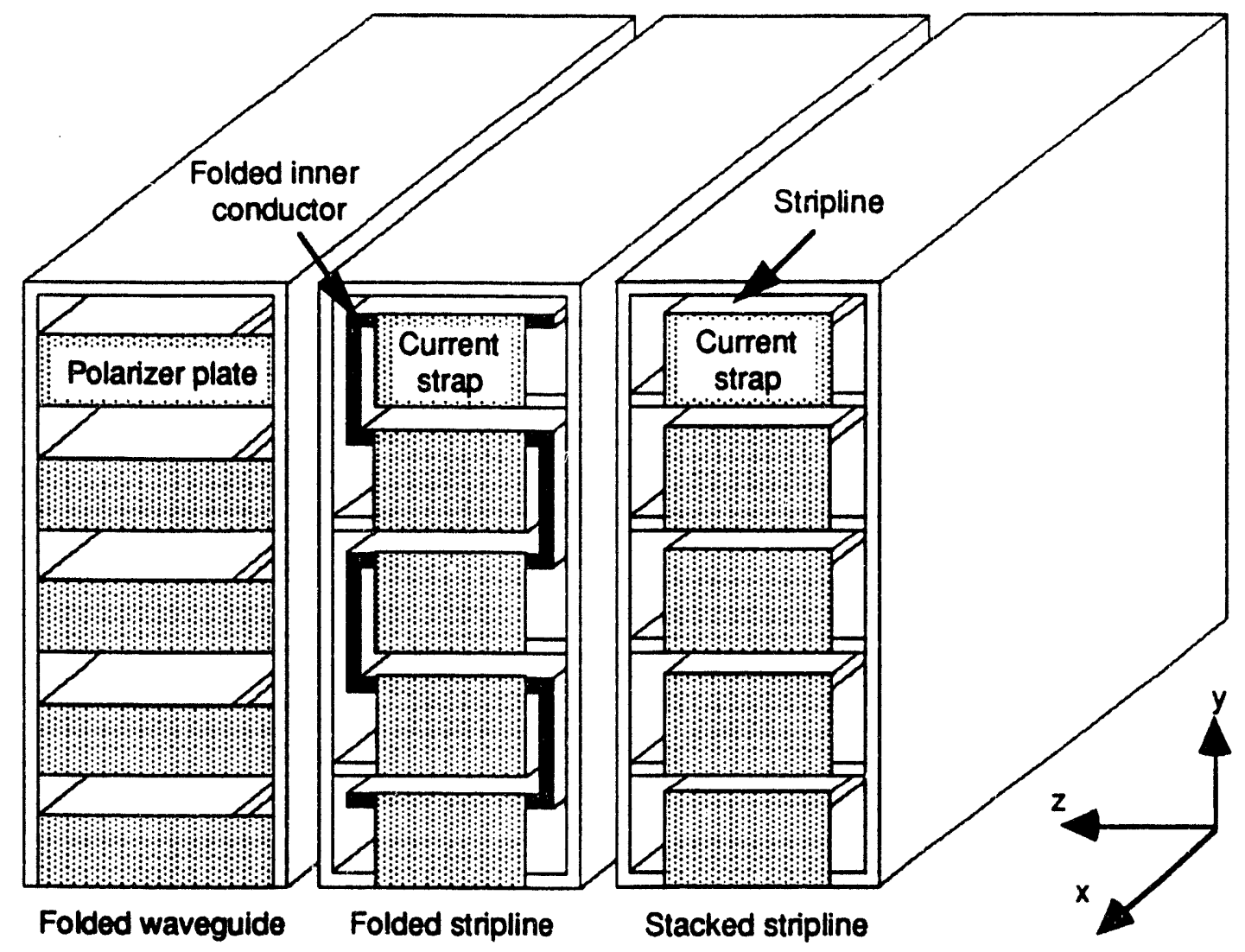

Fig. 1. A side-by-side comparison of the design features of the three resonant cavity concepts.

elements, by a combination of adjustments to cavity length and position of the coaxial line coupler along the inner conductor (tap point adjustment), or by a combination of these. Experience with the folded waveguide suggests that external tuning and matching are preferable as a practical matter.

The concept known as the stacked stripline takes the previous concept a step further by using multiple stripline resonators and current straps, as shown in Fig. 1. Note that each resonator is independent of the others except at the output, where mutual coupling can be significant. This means that it is very important to tune each line separately to the same resonant frequency with the other lines shorted and to match each to the same impedance tap point prior to operating them in a ganged mode. It is also assumed that the input power is divided from a single source, so it is equally important that all of the inputs have the same phase relationship.

\section{RESULTS}

We constructed same-size, small-scale cavities of each concept to ascertain how they compare with each other. Each had outside dimensions of $6.0 \times 14.0 \times 61.3 \mathrm{~cm}$. The output end of each was configured as shown in Fig. 1. All couplers were designed with a sliding tap point to allow freedom of travel in the long dimension for impedance matching. The input signal for the stacked stripline was split using an 
ANZAC DS-312, four-way splitter and equal line lengths to individual stripline connectors. The fifth resonator was undriven but was tuned like the others to provide the best output coupling. Tuning for the stacked stripline was done by flexing the inner conductor of each stripline resonator to slightly change the characteristic impedance along the line until they all resonated at the same frequency. Measurements were taken with an HP 8753C Network Analyzer.

The measurement of importance for this preliminary comparison was the relative magnetic field coupled to a loop probe at the output end of the launcher. The loop probe, $2 \mathrm{~mm}$ in diameter, was positioned to intercept the magnetic flux vector in the $z$-direction (Fig.1). A comparison of scans of relative signal strengths in the $y$-direction is shown in Fig. 2. Both of the stripline concepts seem to have better magnetic flux coupling along the vertical centerline than the folded waveguide. The folded waveguide, in turn, has significantly more flux linkage than loop-type designs. The stacked stripline has the best coupling, though its profile is significantly more peaked. This peaking is primarily due to the nonoptimized geometry for multiply coupled resonators, and the fact that only four of the five resonators are being driven.
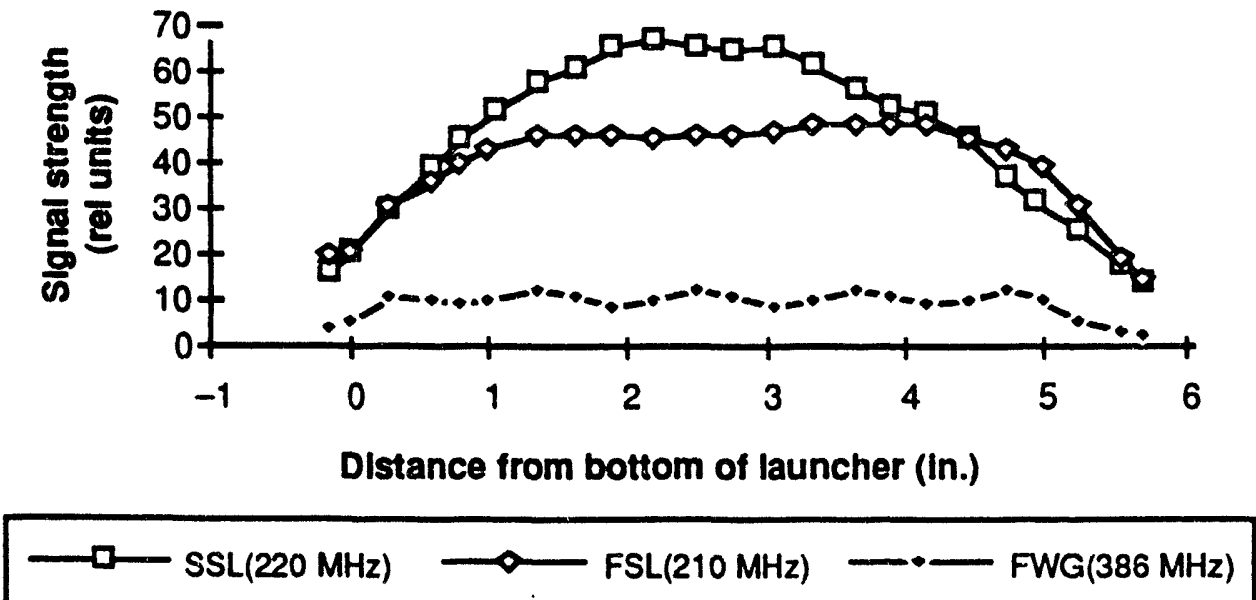

Fig. 2. Vertical scans of the coupled magnetic flux in the z-direction for the stacked stripline (SSL), folded stripline (FSL), and folded waveguide (FWG). Cavity resonant frequencies are also shown.

Horizontal scans were also made for the striplines. A representative scan for the stacked stripline is shown in Fig. 3 and is very similar in form to those taken for the folded stripline case. The structure is very reminiscent of that observed for loop-type launchers, where there are large return currents in the side walls. These currents can be adjusted by slotting the side walls as is done for loop-type launchers.

The features of both stripline designs suggest that they are hybrids of the folded waveguide and loop-type geometries. The key features that make them attractive are the low electric field at the output and the high magnetic flux coupling, which is aided by the fact that several short current straps carry the current rather than the one or two 
long ones used in loop-type designs-i.e., there is very small current droop because the ratio of the physical length to quarte- wavelength is small.

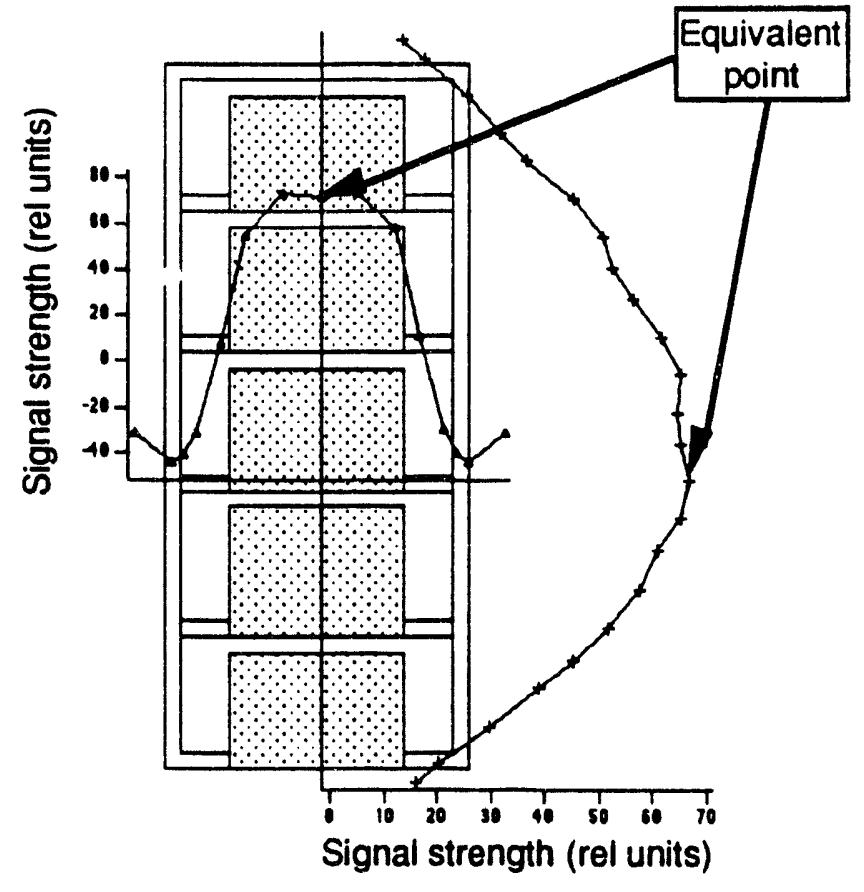

Fig. 3. Vertical and horizontal scans of the stacked stripline coupled magnetic flux. Signai strength for the scan crossing point is also indicated.

\section{REFERENCES}

1. T. L. Owens, IEEE Trans., Plasma Sci., PS-14 (6), 934 (1986).

2. T. L. Owens et al., AIP Conf. Proc. 159: Appl. of RF Power to Plasmas, 298 (1987).

3. G. R. Haste and D. J. Hoffman, AIP Conf. Proc. 190: Appl. of RF Power to Plasmas, 266 (1989).

4. F. W. Baity et al., AIP Conf. Proc. 244: RF Power in Plasmas, 298 (1991).

5. G. R. Haste et al., Fus. Engr. \& Dsgn., accepted for publication. 

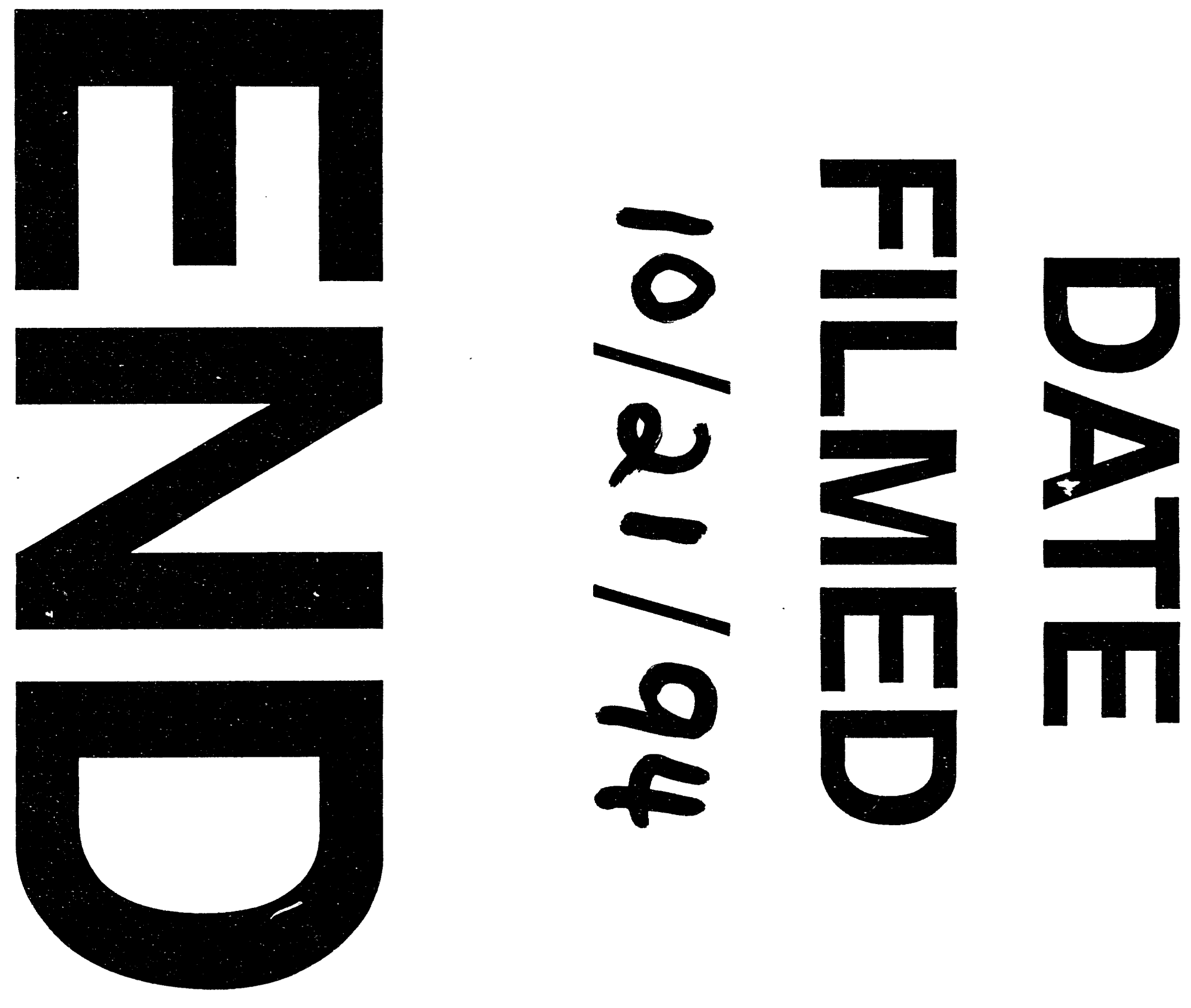
